Deciphering the Activity and Quiescence of High-redshift Cluster Environments : ALMA Observations of Cl J1449+0856

at $z=2$

Strazzullo, V.

2018-07-20

Strazzullo, V, Coogan , R T, Daddi , E, Sargent, M T, Gobat, R, Valentino , F , Bethermin , M , Pannella, M , Dickinson, M , Renzini , A, Arimoto , N , Cimatti , A , Dannerbauer , H , Finoguenov , A, Liu , D \& Onodera , M 2018 , ' Deciphering the Activity and Quiescence of High-redshift Cluster Environments : ALMA Observations of $\mathrm{Cl}$ J1449+0856 at $\mathrm{z}=2{ }^{\prime}$, Astrophysical Journal , vol. 862 , no. 1 , 64 . https://doi.org/10.3847/1538-4357/aacd10

http://hdl.handle.net/10138/238267

https://doi.org/10.3847/1538-4357/aacd10

unspecified

publishedVersion

Downloaded from Helda, University of Helsinki institutional repository.

This is an electronic reprint of the original article.

This reprint may differ from the original in pagination and typographic detail.

Please cite the original version. 


\title{
Deciphering the Activity and Quiescence of High-redshift Cluster Environments: ALMA Observations of Cl J1449+0856 at $z=2$
}

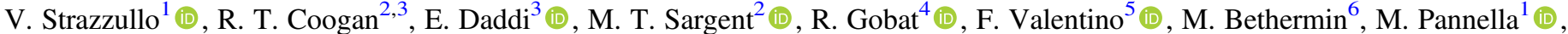

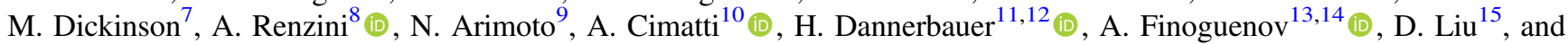 \\ M. Onodera ${ }^{16,17}$ (i) \\ ${ }^{1}$ Department of Physics, Ludwig-Maximilians-Universität, Scheinerstr. 1, D-81679 München, Germany; vstrazz@usm.lmu.de \\ 2 Astronomy Centre, Department of Physics and Astronomy, University of Sussex, Brighton BN1 9QH, UK \\ ${ }^{3}$ CEA, IRFU, DAp, AIM, Université Paris-Saclay, Université Paris Diderot, Sorbonne Paris Citè, CNRS, F-91191 Gif-sur-Yvette, France \\ ${ }^{4}$ Instituto de Física, Pontificia Universidad Católica de Valparaíso, Casilla 4059, Valparaíso, Chile \\ ${ }^{5}$ Dark Cosmology Centre, Niels Bohr Institute, University of Copenhagen, Juliane Maries Vej 30, DK-2100 Copenhagen, Denmark \\ ${ }_{7}^{6}$ Aix Marseille Univ., CNRS, LAM, Laboratoire d'Astrophysique de Marseille, Marseille, France \\ ${ }_{8}^{7}$ National Optical Astronomy Observatory, 950 North Cherry Avenue, Tucson, AZ 85719, USA \\ ${ }^{8}$ INAF-Osservatorio Astronomico di Padova, Vicolo dell'Osservatorio 5, I-35122, Padova, Italy \\ ${ }^{9}$ Astronomy Program, Department of Physics and Astronomy, Seoul National University, 599 Gwanak-ro, Gwanak-gu, Seoul, 151-742, Republic of Korea \\ ${ }^{10}$ Dipartimento di Fisica e Astronomia, Universitá di Bologna, via Gobetti 93/2, I-40129, Bologna, Italy \\ ${ }^{11}$ Instituto de Astrofisica de Canarias (IAC), E-38205 La Laguna, Tenerife, Spain
${ }^{2}$ Universidad de La Laguna, Dpto. Astrofisica, E-38206 La Laguna, Tenerife, Spain \\ ${ }^{13}$ Max-Planck-Institut für extraterrestrische Physik, Giessenbachstrasse 1, D-85748 Garching, Germany \\ ${ }^{14}$ Department of Physics, University of Helsinki, P. O. Box 64, FI-00014, Helsinki, Finland \\ ${ }^{15}$ Max Planck Institute for Astronomy, Konigstuhl 17, D-69117 Heidelberg, Germany \\ ${ }^{16}$ Subaru Telescope, National Astronomical Observatory of Japan, National Institutes of Natural Sciences (NINS), 650 North A'ohoku Place, Hilo, HI, 96720 , USA \\ ${ }_{17}$ Department of Astronomical Science, SOKENDAI (The Graduate University for Advanced Studies), 650 North A'ohoku Place, Hilo, HI 96720, USA \\ Received 2017 November 2; revised 2018 June 8; accepted 2018 June 15; published 2018 July 24
}

\begin{abstract}
We present Atacama Large Millimeter/submillimeter Array observations of the $870 \mu \mathrm{m}$ continuum and CO(4-3) line emission in the core of the galaxy cluster $\mathrm{Cl} \mathrm{J1449+0856}$ at $z=2$, a near-IR-selected, X-ray-detected system in the mass range of typical progenitors of today's massive clusters. The $870 \mu \mathrm{m}$ map reveals six $F_{870 \mu \mathrm{m}}>0.5 \mathrm{mJy}$ sources spread over an area of $0.07 \mathrm{arcmin}^{2}$, giving an overdensity of a factor of $\sim 10(6)$ with respect to blank-field counts down to $F_{870 \mu \mathrm{m}}>1 \mathrm{mJy}(>0.5 \mathrm{mJy})$. On the other hand, deep CO(4-3) followup confirms membership of three of these sources but suggests that the remaining three, including the brightest $870 \mu \mathrm{m}$ sources in the field $\left(F_{870 \mu \mathrm{m}} \gtrsim 2 \mathrm{mJy}\right)$, are likely interlopers. The measurement of $870 \mu \mathrm{m}$ continuum and $\mathrm{CO}(4-3)$ line fluxes at the positions of previously known cluster members provides a deep probe of dusty star formation occurring in the core of this high-redshift structure, adding up to a total star formation rate of $\sim 700 \pm 100 M_{\odot} \mathrm{yr}^{-1}$ and yielding an integrated star formation rate density of $\sim 10^{4} M_{\odot} \mathrm{yr}^{-1} \mathrm{Mpc}^{-3}$, five orders of magnitude larger than in the field at the same epoch, due to the concentration of star-forming galaxies in the small volume of the dense cluster core. The combination of these observations with previously available Hubble Space Telescope imaging highlights the presence in this same volume of a population of galaxies with already suppressed star formation. This diverse composition of galaxy populations in Cl J1449+0856 is especially highlighted at the very cluster center, where a complex assembly of quiescent and star-forming sources is likely forming the future brightest cluster galaxy.
\end{abstract}

Key words: galaxies: clusters: individual (Cl J1449+0856) - galaxies: evolution - galaxies: high-redshift

\section{Introduction}

Thanks to a variety of efforts devoted to the search for distant progenitors of galaxy clusters, pushing toward $z \sim 2$ and beyond (e.g., Rosati et al. 1999; Kurk et al. 2000; Mullis et al. 2005; Stanford et al. 2006, 2012; Venemans et al. 2007; Eisenhardt et al. 2008; Andreon et al. 2009; Wilson et al. 2009; Papovich et al. 2010; Gobat et al. 2011; Santos et al. 2011; Spitler et al. 2012; Muzzin et al. 2013; Wylezalek et al. 2013; Clements et al. 2014; Bleem et al. 2015; Casey et al. 2015; Strazzullo et al. 2015; Flores-Cacho et al. 2016; Wang et al. 2016; Cai et al. 2017; Daddi et al. 2017; Mantz et al. 2018), it has been possible in the past decade to significantly extend the timeline and scope of galaxy evolution studies in the densest high-redshift environments. This has eventually bridged the historically divided cluster and protocluster regimes (see recent review by Overzier 2016), at a cosmic time that is thought-and indeed turns out- to be a transformational epoch for both clusters and their galaxies. The synergy of observations at different wavelengths, including optical/near-IR (NIR) to probe stellar populations and galaxy structural properties, mid/far-IR to radio to probe star formation rates (SFRs), submillimeter for gas reservoirs, and X-ray, mid-IR, and radio for nuclear activity, has proved fundamental in exploring the many facets of cluster galaxy populations, as discussed below.

From observations of massive cluster galaxies at lower redshifts (e.g., Andreon 2006; De Propris et al. 2007; Lidman et al. 2008; Mei et al. 2009; Mancone et al. 2010; Strazzullo et al. 2010; Wylezalek et al. 2014), we expect that the epoch around $z \sim 2$ corresponds to the transition from a regime of widespread, high levels of star formation in dense environments to the quiescent regime characteristic of cluster cores at $z \lesssim 1$. Direct observations at high redshifts have in fact detected increasing levels of star formation, as well as nuclear and merging activity in distant $z \gtrsim 1.5$ groups and clusters 
(e.g., Hayashi et al. 2010, 2011; Hilton et al. 2010; Zeimann et al. 2012; Brodwin et al. 2013; Lotz et al. 2013; Dannerbauer et al. 2014; Ma et al. 2015; Popesso et al. 2015b; Santos et al. 2015; Tran et al. 2015; Alberts et al. 2016; Hung et al. 2016; Wang et al. 2016; Krishnan et al. 2017; Nantais et al. 2017, and references therein). At the same time, passively evolving galaxies are often found to be overrepresented, to different degrees, in the densest regions of these environments (e.g., Steidel et al. 2005; Kurk et al. 2009; Papovich et al. 2010; Snyder et al. 2012; Spitler et al. 2012; Gobat et al. 2013; Kubo et al. 2013; Strazzullo et al. 2013; Tanaka et al. 2013; Hatch et al. 2016), and examples of (generally massive) clusters with already very strongly suppressed star formation are also found up to $z \sim 2$ (e.g., Strazzullo et al. 2010; Andreon et al. 2014; Newman et al. 2014; Cooke et al. 2016). Cluster selection, cluster-to-cluster variation, the intrinsically transitional phase of galaxy populations at this time, and observational difficulties have all contributed to assemble a varied, still unfinished picture that might at times still look controversial in some aspects, and sometimes difficult to reconcile with theoretical expectations (e.g., Granato et al. 2015).

Thanks to expensive-and thus still limited to a relatively small number of systems-dedicated follow-up programs, a number of recent studies have started investigating in more specific detail the properties of both quiescent and star-forming galaxies in distant (proto)cluster environments. Such studies explored a variety of aspects, including the environmental dependence of stellar ages and structure of passive populations (e.g., Gobat et al. 2013; Strazzullo et al. 2013; Tanaka et al. 2013; Andreon et al. 2014, 2016; Newman et al. 2014; Beifiori et al. 2017; Lee-Brown et al. 2017; Prichard et al. 2017; Chan et al. 2018); the environmental dependence of the specific SFRs (sSFRs), metallicities, and dust attenuation properties of starforming galaxies (e.g., Tanaka et al. 2010; Hatch et al. 2011; Hayashi et al. 2011, 2016; Koyama et al. 2013, 2014; Kulas et al. 2013; Cooke et al. 2014; Smail et al. 2014; Kacprzak et al. 2015; Shimakawa et al. 2015, 2018; Tran et al. 2015; Valentino et al. 2015; Husband et al. 2016; Kewley et al. 2016); and the environmental dependence of cold gas reservoirs fueling star formation in dense environments (e.g., Aravena et al. 2012; Casasola et al. 2013; Emonts et al. 2013; Ivison et al. 2013; Tadaki et al. 2014; Casey 2016; Gullberg et al. 2016; Dannerbauer et al. 2017; Hayashi et al. 2017, 2018; Lee et al. 2017; Noble et al. 2017; Rudnick et al. 2017; Stach et al. 2017). Results from such investigations critically shape our understanding of galaxy population properties - and of the processes affecting galaxy evolution-in early dense environments, though the still very limited cluster galaxy samples, small number of clusters probed, and selection biases continue to preclude conclusive interpretations.

We present here new results from Atacama Large Millimeter/submillimeter Array (ALMA) observations of the galaxy cluster Cl J1449+0856 (hereafter Cl J1449; Gobat et al. 2011) at $z=2$, complementing our previous work on its galaxy populations (Gobat et al. 2013; Strazzullo et al. 2013, 2016; Valentino et al. 2015, 2016) with a critical independent vantage point. Cl J1449 was identified as an overdensity of IRAC colorselected galaxies and spectroscopically confirmed with now 30 spectroscopic members (Gobat et al. 2011, 2013; Valentino et al. 2015; Coogan et al. 2018). The estimated halo mass based on its extended X-ray emission and stellar mass content is $(5-7) \times 10^{13} M_{\odot}$ (Valentino et al. 2016, and references therein), placing this structure in the mass range of the average progenitors of today's typical massive clusters. For what directly concerns the results presented here, our previous work has highlighted the mixed galaxy population in this cluster, consisting of both quiescent and highly star-forming sources (as well as active galactic nuclei [AGNs]; Gobat et al. 2013; Strazzullo et al. 2013; Valentino et al. 2015, 2016). In particular, in Strazzullo et al. (2016, hereafter S16) we investigated the nature of the massive red population characterizing the cluster core, in terms of dusty massive star-forming galaxies and sources with already-suppressed star formation, based on a purely photometric analysis at optical/ NIR wavelengths. On the other hand, in spite of the statistical validity of this approach, the ultimate confirmation of the nature of such sources, as well as an actual estimate of the (obscured) star formation occurring in the cluster core, remains with SFR indicators not biased by dust attenuation. Cl J1449 had been previously observed with Spitzer/MIPS $(24 \mu \mathrm{m})$ and later also with Herschel/PACS and SPIRE, which indeed suggested potentially high levels of star formation activity right in the cluster core as already reported in the first study by Gobat et al. (2011). However, the angular resolution and depth of these data, and/or ambiguities with respect to contamination from nuclear activity, hampered the effectiveness of these observations in establishing a reliable picture of star formation and quenching in this system.

In this work based on ALMA observations, we thus focus on three main aspects: (1) the quantification of star formation

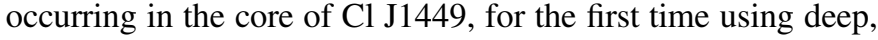
high-resolution star formation probes not biased by dust attenuation (Sections 3 and 4.1); (2) the constraints set by these new observations on the first massive quiescent galaxies that, even as early as $z \sim 2$, are a significant feature of the core of Cl J1449 (Section 4.2); and (3) the direct observation of the forming brightest cluster galaxy (BCG; Section 5), which, especially thanks to the combination with Hubble Space Telescope (HST) data, produces a remarkably detailed picture of a critical phase in the early evolution of (proto-)BCGs. Besides the scope of this paper, the ALMA observations discussed here enable the investigation of a significantly wider range of questions related to the effect of the environment on the properties and evolution of high-redshift cluster galaxies: the companion paper by Coogan et al. (2018, hereafter C18) presents in particular the dust and gas properties of the ALMAdetected cluster members and provides extensive descriptions of all submillimeter and radio observations of $\mathrm{Cl} \mathrm{J} 1449$.

We assume a $\Lambda$ CDM cosmology with $\Omega_{M}=0.3, \Omega_{\Lambda}=0.7$, and $H_{0}=70 \mathrm{~km} \mathrm{~s}^{-1} \mathrm{Mpc}^{-1}$. Stellar masses and SFRs are quoted for a Salpeter (1955) initial mass function (IMF). Magnitudes and colors are quoted in the AB system.

\section{Observations}

The central area of $\mathrm{Cl}$ J1449 was observed in ALMA Cycle 1 and 3 programs 2012.1.00885.S and 2015.1.01355.S. The Cycle 1 program obtained a band 7 mosaic probing $870 \mu \mathrm{m}$ continuum over a $\sim 0.3 \operatorname{arcmin}^{2}$ region in the cluster central area. Observations were completed in 2014 December for a total on-source time of $\sim 2.3 \mathrm{hr}$. The probed field, offset by $8^{\prime \prime}$ from the cluster center, reaches out to clustercentric distances of $\sim 100-200 \mathrm{kpc}$ depending on the direction (see Figure 1). For comparison, the estimated cluster virial radius is 

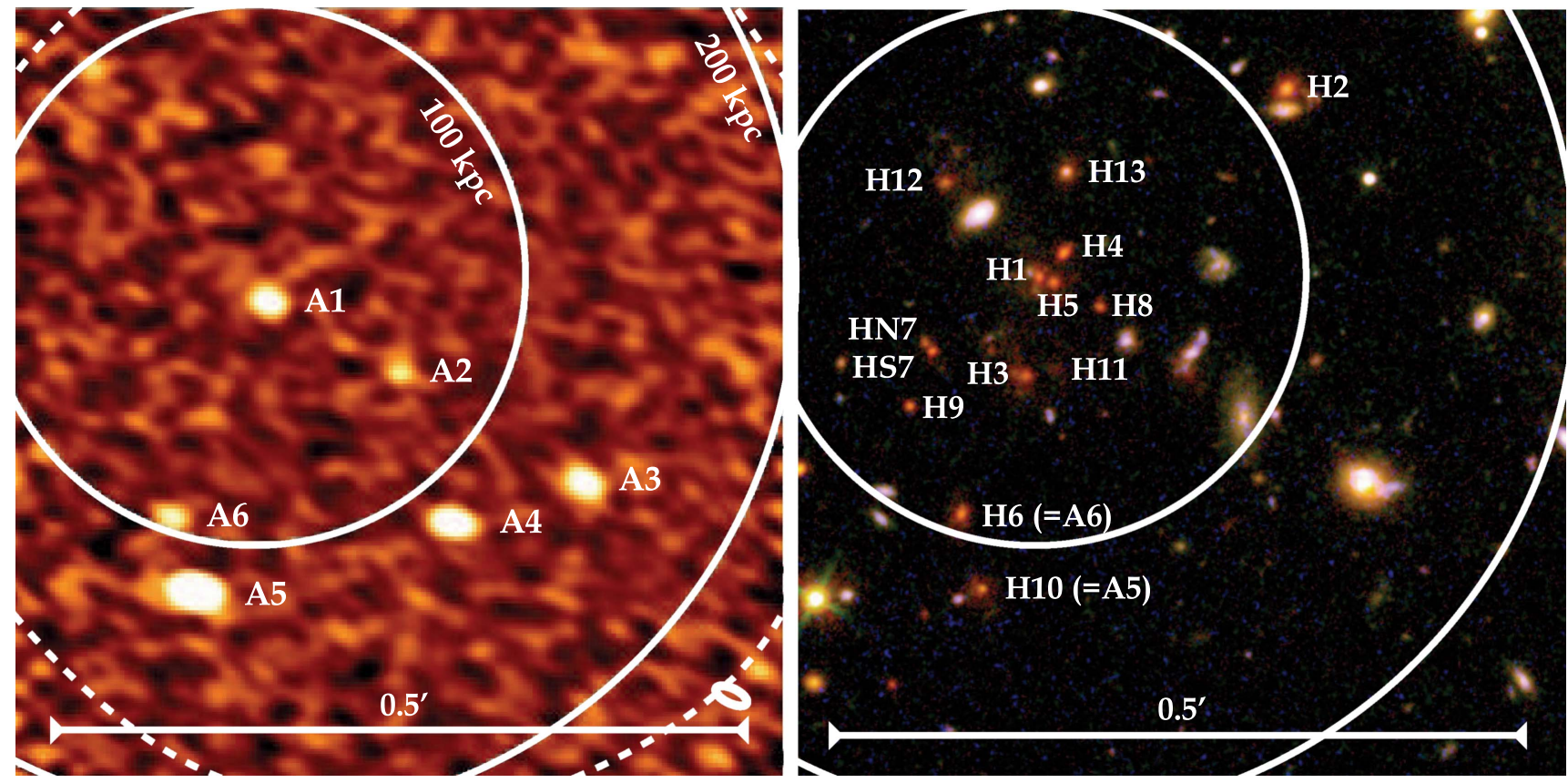

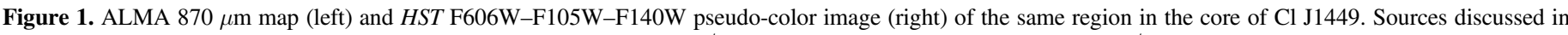

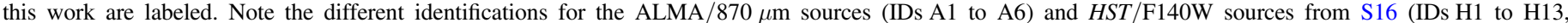

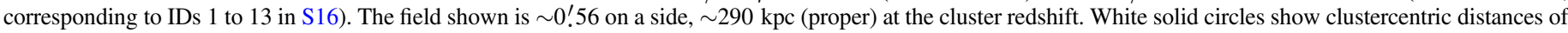

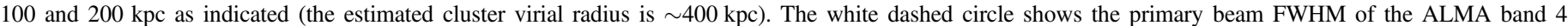
observations.

$r_{200} \sim 0.4 \pm 0.1 \mathrm{Mpc}$ (Gobat et al. 2013; Valentino et al. 2016).

The synthesized beam of FWHM $\sim 1$ !" $4 \times 0$ ". 6 is well suited for the size of $z \sim 2$ galaxies and to avoid confusion in the crowded cluster-core environment. The map has an rms sensitivity of $\sim 70 \mu \mathrm{Jy}_{\text {beam }}{ }^{-1}$ and thus, as a reference, reaches down to a SFR of $\sim 40 M_{\odot} \mathrm{yr}^{-1}$ (3 $\sigma$; Béthermin et al. 2012, see Section 4.1) for "main sequence" (MS; Elbaz et al. 2011) starforming galaxies at $z \sim 2$, corresponding to stellar masses of $\sim 2 \times 10^{10} M_{\odot}$ (Sargent et al. 2014). The same program also obtained a single overlapping pointing in band 3 to probe $\mathrm{CO}(3-2)$ emission from cluster galaxies at matched depth with the $870 \mu \mathrm{m}$ continuum, with the main goals of confirming cluster membership of sources detected in the $870 \mu \mathrm{m}$ map and estimating gas reservoirs fueling their star formation. However, as the later acquired $\mathrm{CO}(4-3)$ observations discussed below are deeper (see C18), and as higher-order CO transitions are better SFR (rather than total molecular gas mass) tracers (e.g., Daddi et al. 2015; Liu et al. 2015), these $\mathrm{CO}(3-2)$ data are not used for the purposes of this work.

The Cycle 3 follow-up observed again this region with a band 4 pointing to probe $\mathrm{CO}(4-3)$ emission from cluster members. Observations were completed in May 2016, for a total on-source time of $\sim 2 \mathrm{hr}$. The FWHM of the primary beam is $\sim 41^{\prime \prime}$, well matched to the $870 \mu \mathrm{m}$ mosaic (Figure 1). The synthesized beam FWHM is $\sim 1$ ". $2 \times 1^{\prime \prime}$. The $\mathrm{rms}$ is $10 \mathrm{mJy} \mathrm{km} \mathrm{s}^{-1}$ over $100 \mathrm{~km} \mathrm{~s}^{-1}$, corresponding to a $3 \sigma$ detection limit of $\sim 35 M_{\odot} \mathrm{yr}^{-1}$ for a MS galaxy at $z=2$ with a CO(4-3) line FWHM of $\sim 400 \mathrm{~km} \mathrm{~s}^{-1}$.

We focus here on the constraints on star formation activity and its suppression in the core region of $\mathrm{Cl} \mathrm{J} 1449$ derived from the observations of $870 \mu \mathrm{m}$ continuum and $\mathrm{CO}(4-3)$ line emission. We refer the reader to $\mathrm{C} 18$ for a full, extensive summary of the ALMA observations and for a detailed description of the measurements of the $870 \mu \mathrm{m}$ continuum and $\mathrm{CO}(4-3)$ line fluxes that we use in this work. Summarizing those aspects most important to the analysis presented here, we note that $\mathrm{C} 18$ detected continuum sources and independently searched for spectral lines in the band 7 and band 4 observations. Then, both $870 \mu \mathrm{m}$ continuum and $\mathrm{CO}(4-3)$ line fluxes were measured at the positions of all these millimeterdetected sources, as well as at the positions of all known cluster members from our previous optical/NIR studies (Gobat et al. 2011, 2013; Strazzullo et al. 2013, 2016; Valentino et al. 2015). All the analysis and results below are based on these measurements as described in C18.

\section{Overdensity of $870 \mu \mathrm{m}$ Sources}

The $870 \mu \mathrm{m}$ map of the core of $\mathrm{Cl} \mathrm{J} 1449$ reveals six continuum sources $(\mathrm{S} / \mathrm{N}>4)$ with $F_{870 \mu \mathrm{m}}>0.5 \mathrm{mJy}$ (Figure 1), giving a projected source density in the $\sim 0.3 \operatorname{arcmin}^{2}$ survey field that is a factor of $\sim 6$ higher than expected from blank-field counts (e.g., $\sim 1.0 \pm 0.8$ sources would be expected from Oteo et al. 2016). In fact, all six sources are concentrated within a circle of $r \sim 0$ ' 15 . Four of the six $870 \mu \mathrm{m}$ sources are brighter than $1 \mathrm{mJy}$, resulting in a projected overdensity of $\sim 10$ $(\sim 0.3 \pm 0.3$ sources expected to this flux limit from Oteo et al. 2016). We note that, as discussed below, the two brightest $870 \mu \mathrm{m}$ sources are likely to be background interlopers. We estimate their flux magnification due to the cluster potential, assuming a spherical Navarro, Frenk \& White (NFW, 1997) halo of mass $6 \times 10^{13} M_{\odot}$ and a concentration in the range $c=1-5$. For the brightest, $\sim 6 \mathrm{mJy}$ source (labeled A5 below), the estimated photometric redshift $z \sim 2.8$ yields a magnification factor of $\sim 12 \%$ (5\%-18\% within the $3 \sigma$ range of the photometric redshift). For the other source (A4, $\sim 1.9 \mathrm{mJy})$, we estimate a magnification of about $9 \%-20 \%(30 \%, 40 \%)$ for a source redshift from 2.5 to $3.5(5,7)$. Even for a source redshift $z \sim 7$, 

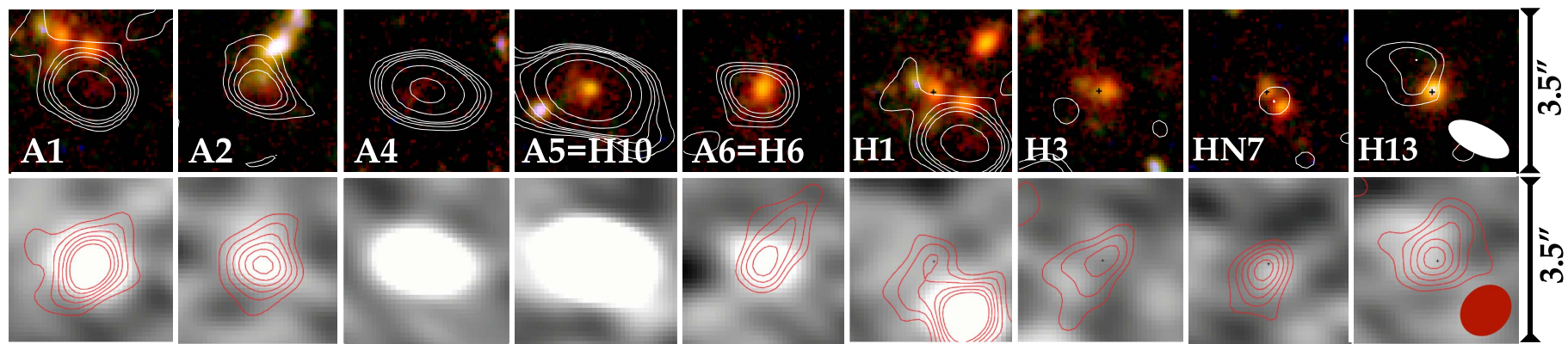

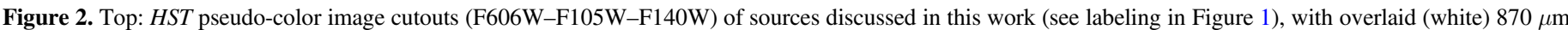

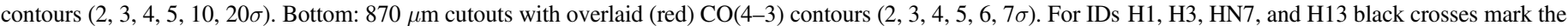
$H S T / F 140 \mathrm{~W}$ galaxy position. White and red ellipses in rightmost panels show the synthesized beam of the $870 \mu \mathrm{m}$ and band 4 observations, respectively.

the flux would still be brighter than $1 \mathrm{mJy}$. We thus conclude that the overdensity of millimeter-bright sources is not likely significantly affected by lensing magnification by the cluster potential.

The left panel of Figure 1 identifies all ALMA $870 \mu \mathrm{m}$ selected sources discussed in this work (labeled A1 to A6), while the right panel identifies the HST-selected (F140W) red sources identified in S16 (labeled H1 to H13; note that H6 and A6-and $\mathrm{H} 10$ and A5-correspond to the same galaxy, as indicated). Of the six $870 \mu \mathrm{m}$ sources, two have spectroscopic redshifts measured in the Gobat et al. (2013) HST grism followup of Cl J1449: a foreground galaxy at $z \sim 1.3$ (A3, thus ignored henceforth), and the cluster member A6 = H6. None of the other $870 \mu \mathrm{m}$ sources have an optical/NIR spectroscopic redshift determination. The $H S T$ counterparts to A1, A2, and A4 are very faint ${ }^{18}$ and were thus not included in our previous studies of galaxy populations in $\mathrm{Cl} \mathrm{J} 1449$. A5 was included in our previous work and was deemed to be likely an interloper at $z_{\text {phot }} \sim 2.8$ (Strazzullo et al. 2013).

On the other hand, A1 and A2 both show a highly significant detection of $\mathrm{CO}(4-3)$ line emission (Figure 2 and $\mathrm{C} 18$ ), securely confirming their cluster membership. However, no lines are detected for the two brightest $870 \mu \mathrm{m}$ sources, A4 and A5. In fact, as discussed at length in $\mathrm{C} 18$, in spite of their high $870 \mu \mathrm{m}$ fluxes, no lines are detected for these sources in any of our data sets probing $\mathrm{CO}(4-3), \mathrm{CO}(3-2)$, and $\mathrm{CO}(1-0)$ at the cluster redshift, as well as bright millimeter lines ([C I](2-1), $\mathrm{C}$ II, $\mathrm{CO}$ transitions up to $\mathrm{CO}(7-6))$ over a significant fraction of the $1<z<9$ range. Nonetheless, several redshifts remain unprobed, notably including the range around the photometric redshift of A5 (C18). Therefore, we do not currently have confirmation of the redshift of A4 and A5. We note that the likelihood of observing two such bright sources unrelated to $\mathrm{Cl}$ J1449 in the small field probed is extremely low: as discussed in more detail in $\mathrm{C} 18$, these sources might in principle still be cluster members with very recently and rapidly suppressed star formation, with the lack of $\mathrm{CO}(4-3)$ emission being potentially reconciled with their bright $870 \mu \mathrm{m}$ continuum by the gas and dust tracing star formation on different timescales. Nonetheless, given their large $870 \mu \mathrm{m}$ fluxes and thus expected very bright CO line emission compared to the depth of our observations (Figure 3), we currently conclude that, at face value, the most

\footnotetext{
${ }^{18} \mathrm{~A} 1$ and A2 are also close to a bright neighbor and were not extracted as individual sources in our F140W-based catalogs (e.g., Strazzullo et al. 2013) until $H S T / \mathrm{F} 105 \mathrm{~W}$-band imaging (S16) and ALMA observations pointed to these sources being separate components.
}

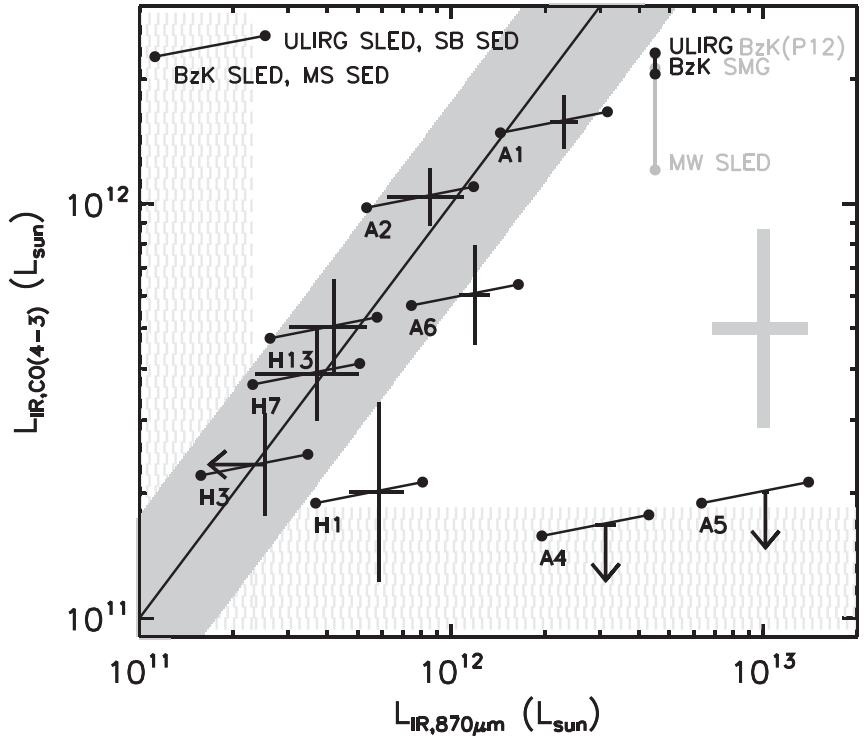

Figure 3. IR luminosities of millimeter-detected sources as estimated from the $870 \mu \mathrm{m}$ continuum fluxes $\left(L_{\mathrm{IR}, 870 \mu \mathrm{m}}\right)$ and from the $\mathrm{CO}(4-3)$ line emission $\left(L_{\mathrm{IR}, \mathrm{CO} 43}\right)$. The double symbol shows $L_{\mathrm{IR}, \mathrm{CO} 43}$ as estimated assuming a BzK or ULIRG CO SLED, and $L_{\mathrm{IR}, 870 \mu \mathrm{m}}$ as estimated with a main-sequence (MS) or starburst (SB) SED, as indicated. For further comparison, the systematics on $L_{\mathrm{IR}, \mathrm{CO} 43}$ corresponding to a range of different assumptions on the CO SLED are shown in the top right corner (see text). Black error bars plotted in the middle of the two estimates show the uncertainties due to the $870 \mu \mathrm{m}$ and $\mathrm{CO}(4-3)$ flux measurements. The black line marks the bisector $\left(L_{\mathrm{IR}, 870 \mu \mathrm{m}}=\right.$ $\left.L_{\mathrm{IR}, \mathrm{CO} 43}\right)$. The thick gray band along the bisector and the gray cross both show the uncertainty deriving from the $(1 \sigma)$ scatter in the adopted scaling relations (see text). Arrows show $2 \sigma$ upper limits (see text). Hatched areas are below the $3 \sigma$ limits for MS/BzK sources (see text). Note the clear outliers A4 and A5, which we therefore conclude are likely interlopers.

likely explanation is that A4 and A5 are interlopers. Among the six bright $870 \mu \mathrm{m}$ sources, only A1, A2, and A6 are thus confirmed to belong to the cluster.

\section{Activity and Quiescence in Cl J1449}

\subsection{Dusty Star Formation in the Cluster Core}

$\mathrm{CO}(4-3)$ emission is detected at $>3 \sigma$ for a total of seven cluster galaxies, including previously known members $(\mathrm{H} 1, \mathrm{H} 3$, H6 = A6, HN7, H13) and those newly confirmed by the detection of the CO line itself (A1, A2; Section 3). All but one of these (H3) also have a $>2.5 \sigma 870 \mu$ m detection (Figures 2, 3). Figure 3 shows the IR luminosities $L_{\mathrm{IR}}$ of these sources as 
estimated from the $870 \mu \mathrm{m}$ continuum flux $\left(L_{\mathrm{IR}, 870 \mu \mathrm{m}}\right)$ or the $\mathrm{CO}(4-3)$ line flux ${ }^{19}\left(L_{\mathrm{IR}, \mathrm{CO} 43}\right)$.

The IR luminosity estimate $L_{\mathrm{IR}, 870 \mu \mathrm{m}}$ was derived from the measured $870 \mu \mathrm{m}$ flux using the average MS and starburst (SB) SEDs from Béthermin et al. (2012) at $z=2$. The $L_{\mathrm{IR}, \mathrm{CO} 43}$ estimate was derived from the measured $\mathrm{CO}(4-3)$ line flux by assuming the $\mathrm{CO}$ spectral line energy distributions (SLEDs) (in particular, the $\mathrm{CO}(5-4) / \mathrm{CO}(4-3)$ line ratio) of ULIRGs ${ }^{20}$ (intended to represent $\mathrm{SBs}$ ) and star-forming BzK galaxies (intended to represent MS sources; from Papadopoulos et al. 2012; Daddi et al. 2015, respectively) and the CO(5-4) versus $L_{\mathrm{IR}}$ relation from Daddi et al. (2015). We consider the adopted assumptions on the $\mathrm{CO}(5-4) / \mathrm{CO}(4-3)$ line ratio as the most appropriate for galaxies in this sample, but we also show for comparison in Figure 3 the effect on the $L_{\mathrm{IR}, \mathrm{CO} 43}$ estimate of a range of different assumptions on the CO SLED, including those measured for the Milky Way (inner region; Fixsen et al. 1999), SMGs (Bothwell et al. 2013), and the Papadopoulos et al. (2012) model (see discussion in Daddi et al. 2015, and references therein). The $\mathrm{CO}(5-4) / \mathrm{CO}(4-3)$ line ratios from these different determinations are affected to different degrees by measurement uncertainties (see the original papers for details), but for what concerns this work we note that the impact of the different plausible line ratios (including their uncertainties) on our $L_{\mathrm{IR}, \mathrm{CO} 43}$ estimate is clearly subdominant with respect to the scatter in the $\mathrm{CO}(5-4)$ versus $L_{\mathrm{IR}}$ relation, as well as to the typical measurement errors of $\mathrm{CO}(4-3)$ line fluxes in this work.

Figure 3 shows these $L_{\mathrm{IR}, 870 \mu \mathrm{m}}$ and $L_{\mathrm{IR}, \mathrm{CO} 43}$ estimates and the related uncertainties as follows. Black error bars show statistical uncertainties from flux measurement errors. The double symbol adopted for all sources highlights the systematic uncertainties in estimating IR luminosities from the $870 \mu \mathrm{m}$ continuum assuming a MS or SB SED, or from $\mathrm{CO}(4-3)$ line fluxes assuming the BzK or ULIRG SLED, as indicated. The thick gray error bar and shaded area along the bisector show the estimated intrinsic scatter of the adopted scaling relations, that is, the scatter in SED shape (dust temperature) and in the $\mathrm{CO}(5-4)$ versus $L_{\mathrm{IR}}$ relation (concerning $L_{\mathrm{IR}, 870 \mu \mathrm{m}}$ and $L_{\mathrm{IR}, \mathrm{CO} 43}$, respectively). Hatched regions in the figure mark IR luminosities below a corresponding reference $3 \sigma$ limit estimated by assuming the following: for $L_{\mathrm{IR}, 870 \mu \mathrm{m}}$, the $3 \sigma$ limit of the $870 \mu \mathrm{m}$ map (Section 2) and the MS SED; and for $L_{\mathrm{IR}, \mathrm{CO} 43}$, the $3 \sigma$ limit of the band 4 observations at field center (Section 2), a line width of $400 \mathrm{~km} \mathrm{~s}^{-1}$, the BzK $\mathrm{CO}(5-4) / \mathrm{CO}(4-3)$ line ratio, and the $\mathrm{CO}(5-4)$ versus $L_{\mathrm{IR}}$ relation. While these are shown as an indication, the measurements, errors, and upper limits shown for the individual sources account for their actual position within the band 4 primary beam FWHM and highlight the systematics due to the SED or CO SLED choice as discussed, though upper limits for $L_{\mathrm{IR}, \mathrm{CO} 43}$ still assume a line width of $400 \mathrm{~km} \mathrm{~s}^{-1}$.

As Figure 3 shows, the $L_{\mathrm{IR}, \mathrm{CO} 43}$ and $L_{\mathrm{IR}, 870 \mu \mathrm{m}}$ IR luminosity estimates are typically consistent within the estimated uncertainties. The two obvious exceptions are A4 and A5, which

\footnotetext{
19 We use $\mathrm{CO}(4-3)$ line fluxes corrected for flux boosting as detailed in C18. ${ }^{20}$ Given the significant uncertainties on the average $\mathrm{CO}(5-4)$ and $\mathrm{CO}(4-3)$ line fluxes adopted for ULIRGs (Daddi et al. 2015), the $\mathrm{CO}(5-4) / \mathrm{CO}(4-3)$ line ratio used here is based on a weighted fit of the ULIRGs' SLED from $\mathrm{CO}(3-2)$ to $\mathrm{CO}(5-4)$ rather than on the actual ratio of $\mathrm{CO}(5-4)$ and $\mathrm{CO}(4-3)$ line fluxes. As discussed, this ULIRG's line ratio is shown-for comparison with the BzK line ratio-as an indication of the impact of the adopted CO SLED, and the exact value adopted does not have significant effects on the results of this work.
}

both have high $L_{\mathrm{IR}, 870 \mu \mathrm{m}}$ from the bright $870 \mu \mathrm{m}$ flux but no $\mathrm{CO}$ emission, leading to an inconsistent upper limit on $L_{\mathrm{IR}, \mathrm{CO} 43}$ even when accounting for the estimated uncertainties. As discussed above, we therefore conclude that these sources are in fact interlopers. For all other sources, the consistency of $L_{\mathrm{IR}, 870 \mu \mathrm{m}}$ and $L_{\mathrm{IR}, \mathrm{CO} 43}$, besides ensuring cluster membership of the $870 \mu \mathrm{m}$ detections, also confirms the reliability of the SFR estimates.

Summing the derived infrared luminosities of cluster members within the probed $\sim 0.08 \mathrm{Mpc}^{2}$ (proper, at $z=2$ ) region yields a total $L_{\mathrm{IR}} \sim(4.3 \pm 0.5) \times 10^{12} L_{\odot}$ (the error corresponding to the range obtained from $L_{\mathrm{IR}, \mathrm{CO} 43}$ with both ULIRG and BzK SLEDs, and $L_{\mathrm{IR}, 870 \mu \mathrm{m}}$ with a MS SED ${ }^{21}$ ), corresponding to a total SFR of $\sim 700 \pm 100 M_{\odot} \mathrm{yr}^{-1}$ (adopting the Kennicutt [1998] calibration). This yields an overall projected SFR density of $\sim(0.9 \pm 0.1) \times 10^{4} M_{\odot} \mathrm{yr}^{-1} \mathrm{Mpc}^{-2}$ and a SFR volume density of $\sim(1.0 \pm 0.1) \times 10^{4} M_{\odot} \mathrm{yr}^{-1} \mathrm{Mpc}^{-3}$ within the probed region ${ }^{22}$ (over the probed fraction of the virial volume, given the estimated cluster virial radius and assuming that the cluster is spherical). Again, these estimates assume that the two brightest $870 \mu \mathrm{m}$ sources A4 and A5 are interlopers: A5 for itself would otherwise contribute a SFR $\sim 1000 M_{\odot} \mathrm{yr}^{-1}$.

The total unobscured SFR of the ALMA-detected cluster members as estimated from the rest-frame UV luminosity $L_{\mathrm{UV}}$ is $<20 M_{\odot} \mathrm{yr}^{-1}$. Given the SFR threshold reached by these observations, the high $L_{\mathrm{IR}} / L_{\mathrm{UV}}$ of the resulting ALMAdetected sample further highlights how galaxy populations in this cluster core are unusually skewed toward very reddened sources (see also Figure 2; further discussion in Section 4.1.1 and in C18). For comparison, the total unobscured SFR of all cluster galaxies within the same region is estimated to be in the range $100 \pm 20 M_{\odot} \mathrm{yr}^{-1}$, after correcting for incompleteness using the field UV luminosity functions from Parsa et al. (2016) and Alavi et al. (2016).

The measured SFR density is obviously orders of magnitude higher than the field average at the same redshift (e.g., Madau \& Dickinson 2014), as observed in various kinds of other high-redshift structures (e.g., Clements et al. 2014; Dannerbauer et al. 2014; Santos et al. 2015; Tran et al. 2015; Wang et al. 2016). This is in fact largely due to the concentration of (star-forming) galaxies within the small volume of the dense cluster core, rather than to individual galaxies having particularly high SFRs. In fact, the overall sSFR in the ALMA-probed field is $\sim 1.1 \pm 0.6 \mathrm{Gyr}^{-1}$, compared to a field average at $z=2$ of $\sim 1.9_{-0.9}^{+1.9} \mathrm{Gyr}^{-1}$ (Madau \& Dickinson 2014). As shown below (Figure 5) and also discussed in C18, the SFRs of individual sources are generally consistent with MS levels, with the possible exception of the two brightest sources A1 and A2 having higher SFRs.

By comparison with the cluster mass $M_{\text {halo }} \sim(5-7)$ $\times 10^{13} M_{\odot}$, the estimated $L_{\mathrm{IR}}$-derived SFR within the probed volume gives a lower limit (that is, not correcting for the part of the virial volume left unprobed by our ALMA observations) to the total SFR density SFR $/ M_{\text {halo }}$ of $\sim 1300 \pm 400 M_{\odot} \mathrm{yr}^{-1} / 10^{14} M_{\odot}$, after a small correction of the total IR luminosity for the $>3 \sigma \quad L_{\mathrm{IR}, 870 \mu \mathrm{m}}$ sample down to $L_{\mathrm{IR}}=10^{7} L_{\odot}$ assuming the Popesso et al. (2015a) group luminosity function at $z \sim 1.6$,

\footnotetext{
${ }^{21}$ Up to $\sim 8 \times 10^{12} L_{\odot}$ from $L_{\mathrm{IR}, 870 \mu \mathrm{m}}$ if assuming an SB SED for all sources, corresponding to a total SFR of $\sim 1400 M_{\odot} \mathrm{yr}^{-1}$.

${ }^{22} \mathrm{Up}$ to $\sim 1.7 \times 10^{4} M_{\odot} \mathrm{yr}^{-1} \mathrm{Mpc}^{-2}$ and $\sim 2 \times 10^{4} M_{\odot} \mathrm{yr}^{-1} \mathrm{Mpc}^{-3}$ from $L_{\mathrm{IR}, 870 \mu \mathrm{m}}$ if assuming a SB SED for all sources.
} 
which should be the most appropriate for this system (see discussion in Popesso et al. 2015a). At face value, this is in line with the Popesso et al. (2015b) prediction at $z=2$ for massive groups, although we remind that this is a lower limit and it is currently not possible to reliably estimate the overall contribution of the cluster outskirts (we note though that, in the available observations, essentially all the measured IR luminosity is contributed by the very central cluster region, $r \lesssim 100 \mathrm{kpc}$; see Figure 1). The derived lower limit to $\mathrm{SFR} / M_{\text {halo }}$ lies at the upper edge of the Alberts et al. (2016) measurements at $z \sim 1.4$ (accounting for the different IMF and marginal correction to the same $L_{\mathrm{IR}}$ limit), in agreement with the expected further increase out to $z=2$, though we also note that the Alberts et al. (2016) clusters have larger estimated halo masses in the range $(2-5) \times 10^{14} M_{\odot}$ and thus are expected to have lower SFR $/ M_{\text {halo }}$ (e.g., Webb et al. 2013; Popesso et al. 2015b). Indeed, results from the lower halo mass sample of Alberts et al. (2014) would give significantly higher $\mathrm{SFR} / M_{\text {halo }}$ in the same redshift range (see discussion in Alberts et al. 2016), also higher than our lower limit measured here. Similarly, although our lower limit tends to be higher than measurements by, e.g., Smail et al. (2014), Ma et al. (2015), and Santos et al. (2015) on clusters of very different masses $\left(8 \times 10^{13} M_{\odot}\right.$ to $5 \times$ $\left.10^{14} M_{\odot}\right)$ at $z \sim 1.5-1.6\left(\mathrm{SFR} / M_{\text {halo }}\right.$ overall in the range $\left.\sim 500-1000 M_{\odot} \mathrm{yr}^{-1} / 10^{14} M_{\odot}\right)$, it would be fully in line with these measurements for a SFR density evolution similar to that predicted by, e.g., Geach et al. (2006). For comparison, the $\sim 3400 M_{\odot} \mathrm{yr}^{-1}$ observed within the $80 \mathrm{kpc}$ core of the similarly massive $\left(M_{\text {halo }} \sim 8 \times 10^{13} M_{\odot}\right)$ Wang et al. (2016) cluster at $z=2.5$ result in a lower limit $\mathrm{SFR} / M_{\text {halo }}>$ $4000 M_{\odot} \mathrm{yr}^{-1} / 10^{14} M_{\odot}$.

\subsubsection{Color Distribution of ALMA-detected Cluster Galaxies}

Despite the poor statistics due to the very small field probed and relatively small number of massive star-forming cluster members, the ALMA-detected sample in this region appears unusually skewed toward very red (F105W-F140W, dustuncorrected) sources. We show in Figure 4 (bottom panel) the color distribution of subsamples of the ALMA-detected sources in Cl J1449 with different stellar mass and SFR thresholds, as indicated. The adopted stellar mass thresholds $\log \left(M / M_{\odot}\right)=$ 10.1 and 10.5 correspond to the lowest mass of the ALMAdetected cluster members and to a mass above the mass completeness limit of the S16 sample, where our formal $3 \sigma$ limit on $\mathrm{CO}(4-3)$ based SFR probes essentially all of the $1 \sigma$ range of the MS (Figure 5). The adopted SFR thresholds correspond to the nominal $3 \sigma$ and $5 \sigma$ limits of the $\mathrm{CO}(4-3)$ observations in the assumptions discussed in Section 4.1.

Figure 4 (top panel) shows for comparison the color distribution of field galaxies at $z_{\text {phot }}=2 \pm 0.3$ with the same stellar mass and SFR limits, from a control field in GOODS-S. The control field is the same as used in S16, and the adopted measurements are described there in more detail (e.g., their Section 2). We briefly remind here that we used the Guo et al. (2013) photometry, as well as stellar masses, photometric redshifts, and model SEDs from Schreiber et al. (2015) and Pannella et al. (2015). We note that both stellar mass and SFR estimates are derived from SED fitting for the field samples, while we use the $\mathrm{CO}(4-3)$ based SFRs for cluster galaxies. The selection of the field comparison samples cannot thus be considered as properly equivalent to the selection of the
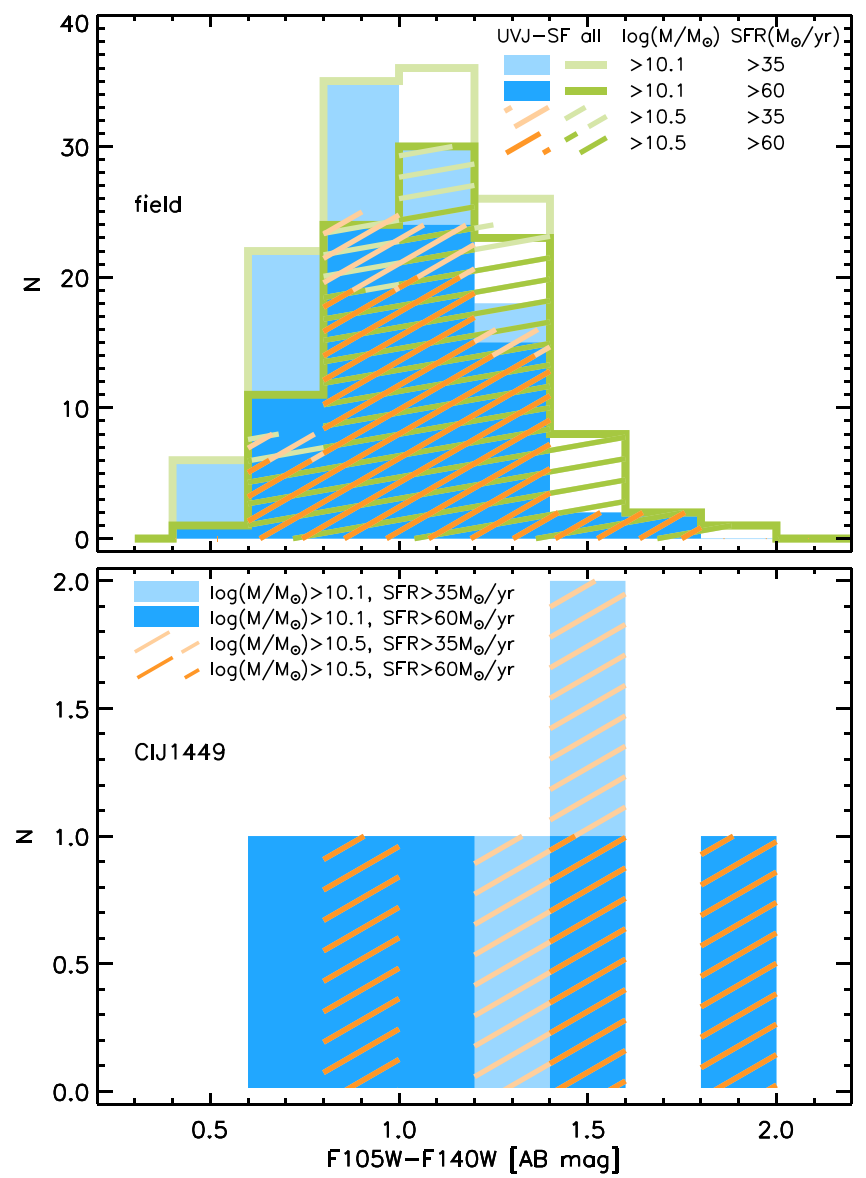

Figure 4. Color distribution of stellar-mass- and SFR-selected samples (with different thresholds as indicated) in a control field (GOODS-S, top panel) and in the cluster field probed by ALMA observations (bottom panel). Note that SFRs for cluster members are based on $\mathrm{CO}(4-3)$ line emission, while the SFRs for the field comparison samples are derived from SED fitting. The green and blue/orange histograms in the top panel refer to field samples including all galaxies and only $U V J$-star-forming sources, respectively (see text).

cluster samples, because of the initial field sample selection (Guo et al. 2013) and the obvious biases between the different (CO(4-3) vs. SED-based) SFR estimates adopted. In this respect, we further note that for the purpose of estimating SFRs for the field sample, a constant star formation history was assumed (model SEDs synthesized with Bruzual \& Charlot 2003) for all sources, allowing for a wide dust attenuation range $\left(A_{v}=0-6\right.$, assuming the Calzetti et al. [2000] attenuation law). SED-based SFRs derived from this modeling have been shown to agree with $L_{\mathrm{IR}}$-based estimates within a $\sim 0.2$ dex scatter (e.g., Pannella et al. 2015, for the same SED analysis as used here).

The green and blue/orange histograms in Figure 4 (top panel) refer to, respectively, samples including all galaxies or only galaxies classified as star-forming based on their restframe UVJ colors (Williams et al. 2009). While the blue/ orange histograms are thus the main reference for the expected color distribution, the green histograms are shown for comparison to account for misclassification of dusty starforming galaxies as quiescent sources; as the bulk of $U V J$ quiescent galaxies are expected to be actually quiescent, this is a conservative comparison sample in this respect, as it maximizes the fraction of red sources. 

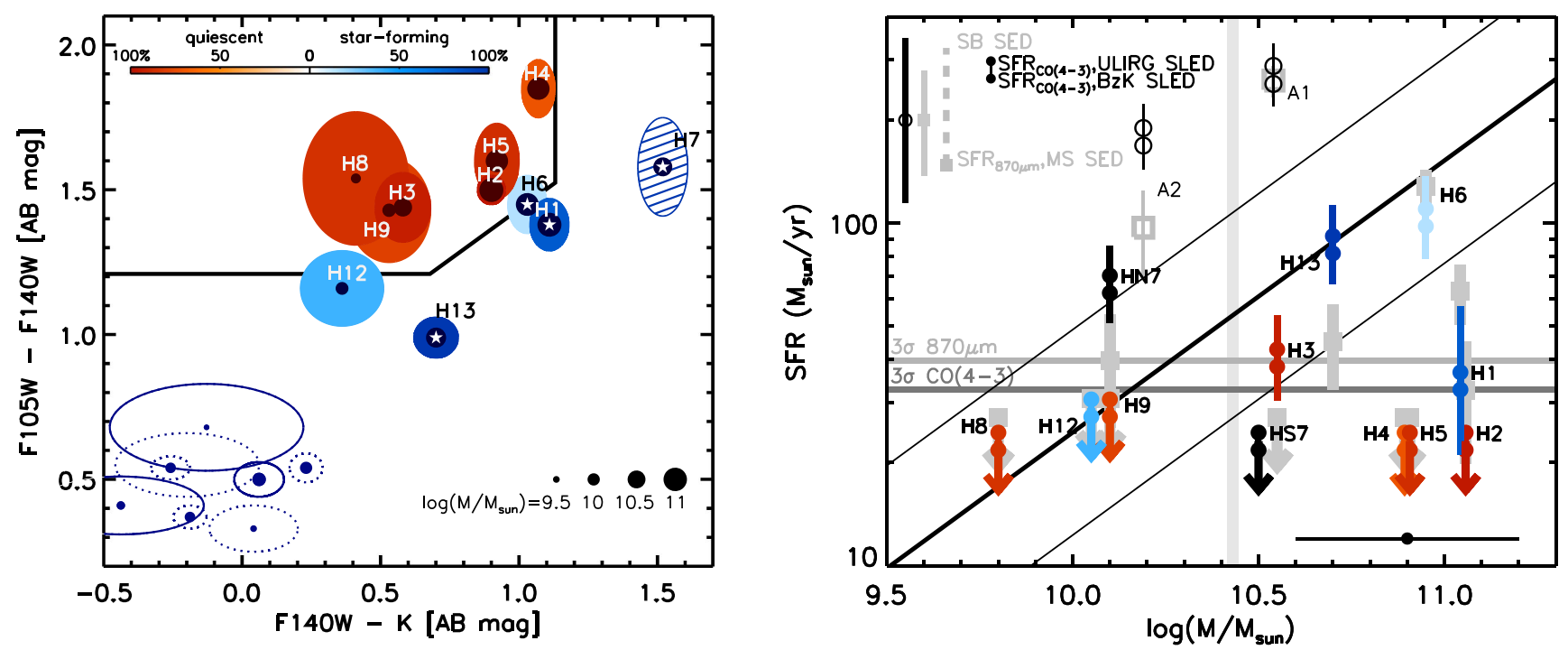

Figure 5. ALMA view of red cluster galaxies. Left: color classification (F140W- $K$ vs./F105W-F140W) of the red galaxy sample as defined in S16. Galaxies are shown with blue or red circles (circle size scales with stellar mass as indicated) according to the classification as star-forming or quiescent, respectively. The (Gaussian) probability of the given classification for each source (see color bar) is defined from photometric uncertainties (shown as ellipses) and does not include uncertainties on the definition of the color selection. Filled labeled ellipses show galaxies in S16's red sample. H7 shows the combined colors of HN7 and HS7 (see text). White stars mark $870 \mu \mathrm{m}$ detected sources. For reference, blue spectroscopic cluster members are also shown as open ellipses (solid or dotted for sources in or outside the ALMA $870 \mu \mathrm{m}$ field, respectively). Right: the stellar mass vs. SFR as determined from $L_{\text {IR.CO43 }}$ (circles). Color-coding in shades of red (blue) reflects the reliability of the classification as quiescent (star-forming) according to the color bar in the left panel. The SFR CO43 $_{3}$ assuming both BzK and ULIRG SLEDs is shown, as in Figure 3. Gray squares show for comparison the SFR as determined from $L_{\mathrm{IR}, 870 \mu \mathrm{m}}$ with a MS SED (the dashed line at the top left shows the effect of using an SB SED). Open symbols show the ALMA-detected sources A1 and A2 (see text). Arrows show $2 \sigma$ upper limits as in Figure 3 . The black and gray error bars at the top left show the uncertainties due to the scatter in the adopted scaling relations for $L_{\mathrm{IR}, \mathrm{CO} 43}$ and $L_{\mathrm{IR}, 870 \mu \mathrm{m}}$, respectively (see Figure 3 ). The black error bar at the bottom right shows the typical uncertainty on stellar masses. The black thick and thin lines show the MS at the cluster redshift (parameterized as in Sargent et al. 2014) and its estimated $(1 \sigma)$ scatter. The horizontal lines show the $3 \sigma$ limits on SFRs inferred from the $870 \mu \mathrm{m}$ and $\mathrm{CO}(4-3)$ line emission, as indicated (see text). The vertical line shows the mass completeness limit of the S16 sample, given the F140W magnitude threshold $\mathrm{m} 140=24.7$, assuming a solar-metallicity simple stellar population (SSP) with no dust attenuation formed at $z \sim 7$.

We show Figure 4 as an indication of our rough expectations for the colors of a mass- and SFR-selected sample of (field) galaxies at the cluster redshift. Considering 1000 realizations of galaxy samples of the same size as the cluster samples shown in Figure 4, randomly drawn from the corresponding field distribution, returns a fraction of F105-F140 $>1.3$ sources larger than that measured in the cluster samples in $<2 \%$ of the realizations at worse (for the $\log \left(M / M_{\odot}\right)>10.1$, SFR $>$ $60 M_{\odot} \mathrm{yr}^{-1}$, or generally $\left.<0.5 \%\right){ }^{23}$ In spite of the caveats outlined above deriving from the nonequivalent selection of the cluster and field samples due to the different adopted SFR indicators, and of the very small number statistics of the ALMA-detected sample in the cluster, the comparison with the first-order expectations from the field sample suggests at face value that very obscured sources are more prevalent than in the field. If confirmed, this would point toward environmental effects possibly related to merger-driven star formation episodes (see, e.g., C18 and references therein) and/ or differences in star formation histories in the cluster environment.

\subsection{The ALMA View of the Red Cluster Galaxy Population}

In S16 we investigated the optically red (F105-F140 $\gtrsim 1)$ cluster galaxy population trying to define its nature, thus probing quenching of star formation and the early appearance

\footnotetext{
${ }^{23}$ Even considering all the $U V J$-quiescent sources as misclassified dusty starforming galaxies, the probability to observe red fractions as high as in the cluster samples in the corresponding samples drawn from the field distributions remains $<2 \%$ (or $<7 \%$ for the $\log \left(M / M_{\odot}\right)>10.1$, SFR $>60 M_{\odot} \mathrm{yr}^{-1}$ sample).
}

of the red sequence in dense environments. Note that this red sample is not a red sequence sample (see S16), as it includes galaxies bluer or redder than the red sequence that are redder than the bulk of the blue star-forming cluster galaxy population. As discussed in S16, cluster galaxies with red (dust-uncorrected) optical colors (F105-F140, $\sim U-B$ rest frame) can obviously be quiescent sources with evolved stellar populations, quenching galaxies with still relatively young stellar populations, or massive dusty star-forming galaxies. Given the characteristics of the available data, in S16 we adopted a star-forming versus quiescent classification based on two broadband colors approximately probing the rest-frame $U-B$ and $B-I$ (Figure 5, left panel; see S16). Although this selection statistically allows the identification of quiescent versus dusty star-forming galaxies, it is still likely to be affected by uncertainties and systematics on an object-byobject basis.

The ALMA observations presented here offer a fully independent view of the red population. Figure 5 shows the ALMA constraints on SFR versus stellar mass for the S16 red sample. The stellar masses shown for this sample are generally SED-based estimates from S16, with the exception of HN7 and HS7, for which most of the photometry used in S16 is expected to be affected by contamination given their very small angular separation. We thus use here stellar mass estimates derived from just the high-resolution F105W and F140W photometry, by empirical calibration on a sample of galaxies in a similar magnitude and redshift range from the GOODS-S control field (as introduced in Section 4.1.1). SFR estimates are derived from the $870 \mu \mathrm{m}$ continuum and $\mathrm{CO}(4-3)$ line emission as discussed in Section 4.1. Figure 5 shows for reference 
(horizontal lines) the $3 \sigma$ limits on such SFRs corresponding to the limits on $L_{\mathrm{IR}, \mathrm{CO} 43}$ and $L_{\mathrm{IR}, 870 \mu \mathrm{m}}$ discussed in Section 4.1, Figure 3. Also here, these are shown as an indication, while the measurements, errors, and upper limits for the individual sources account for the position within the band 4 primary beam, and for the systematics due to the SED or CO SLED choice (upper limits for $\mathrm{SFR}_{\mathrm{CO} 43}$ still assume a line width of $400 \mathrm{~km} \mathrm{~s}^{-1}$ ).

Given the depth of the ALMA observations, the SFR versus stellar mass constraints are mostly effective at high stellar masses, ${ }^{24} \log \left(M / M_{\odot}\right)>10.5$. In this mass range, SFR upper limits suggest a confirmation of the quiescent picture for most sources color-classified as passive, H2, H4, and H5. ${ }^{25}$ The only exception is $\mathrm{H} 3$, which is color-classified as quiescent; it is undetected at $870 \mu \mathrm{m}$ but has a $4.8 \sigma \mathrm{CO}(4-3)$ detection. Given the estimated uncertainties and scatter in the adopted scaling relations (Figures 3,5) the measurements are still consistent with a MS SFR for this source. On the other hand, given the complex morphology and surroundings of this galaxy, as well as the limited resolution of our ALMA observations, it is possible that the "quiescent colors" and the $\mathrm{CO}(4-3)$ line belong to different components.

Concerning instead cluster members in this "red" sample color-classified as star-forming, they are all (but see $\mathrm{H} 7$ below) detected at both $870 \mu \mathrm{m}$ and $\mathrm{CO}(4-3)$. Note that the population of lower-mass $\left(\log \left(M / M_{\odot}\right) \lesssim 10.3\right)$ blue star-forming cluster galaxies (Gobat et al. 2013, S16) is not in general expected to be detected (even when falling within the probed field of view) given the SFR limits shown in Figure 5. The AGN host (Gobat et al. 2013) H13 is consistent with being a MS star-forming galaxy. H1 and $\mathrm{H} 6$ had a particularly uncertain color classification (see Figure 5, left panel, and S16), and indeed H6 was classified as quiescent based on previous SED (Strazzullo et al. 2013) and spectral (Gobat et al. 2013) modeling. Although we are investigating other interpretations for H6 (e.g., Gobat et al. 2018) involving a quiescent component, its SFR estimates from the observed $870 \mu \mathrm{m}$ continuum and $\mathrm{CO}(4-3)$ lime emission are fully consistent with each other, and in line with MS expectations, suggesting that H6 is likely a MS galaxy. On the other hand, Figure 5 suggests that the SFR of H1 might be below the MS scatter. Also note that, although we do our best in dealing with close neighbors by modeling such sources simultaneously when measuring both $870 \mu \mathrm{m}$ and $\mathrm{CO}(4-3)$ fluxes (see $\mathrm{C} 18$ ), the continuum and line fluxes of $\mathrm{H} 1$ are likely affected (to different extents) by residual contamination from neighboring sources and in particular the very bright A1 (see Figure 2), which might possibly explain also the relatively large $L_{\mathrm{IR}, 870 \mu \mathrm{m}}$ versus $L_{\mathrm{IR}, \mathrm{CO} 43}$ offset for this source in Figure 3.

The right panel of Figure 5 also shows the two components HN7 and HS7 of a very close pair hosting an AGN (Gobat et al. 2013). In Figures 3 and 5 (left panels; S16) they are shown as a single source $\mathrm{H} 7$ because the angular resolution attained does not permit deblending of the two components. Given the shape and size of the synthesized $870 \mu \mathrm{m}$ beam, the low $\mathrm{S} / \mathrm{N}$ of the $870 \mu \mathrm{m}$ detection, and the angular distance between the two components, it is not possible to measure the

\footnotetext{
${ }^{24}$ Less massive sources, e.g., H8, H9, H12, would not be detected in our observations even if they were MS star-forming galaxies.

${ }^{25} \mathrm{H} 2$ might have a tentative, low-significance $870 \mu \mathrm{m}$ detection with no associated $\mathrm{CO}(4-3)$ emission (see $\mathrm{C} 18$ and Figure 5), placing it anyway below the MS scatter.
}

$870 \mu \mathrm{m}$ flux of the two components separately. On the other hand, the position of the higher-S/N CO(4-3) detection could suggest that HN7 provides the dominant contribution to the observed CO emission (see HN7-related panels in Figure 2). More importantly, given the redshift difference of the two components, the weak tentative detection of $\mathrm{CO}(4-3)$ line emission of HS7 ( $2 \sigma$; see C18) has a relatively large separation in frequency $(\sim 0.7 \mathrm{GHz})$ from the much stronger detection of HN7, which allows us to separate the respective $\mathrm{CO}(4-3)$ contributions (see $\mathrm{C} 18$ for full details). Given that the total (HN7+HS7) $870 \mu \mathrm{m}$ derived IR luminosity $L_{\mathrm{IR}, 870 \mu \mathrm{m}}$ is fully consistent with the $\mathrm{CO}(4-3)$ based estimate $L_{\mathrm{IR}, \mathrm{CO} 43}$ measured at the position and redshift of HN7 (Figure 3), we conclude that HN7 provides the largest contribution to the measured $870 \mu \mathrm{m}$ flux, with the contamination from HS7 being very likely negligible. The SFR estimated for HN7 is consistent with the expected MS level (Figure 5). The faintness of HS7 would suggest a SFR below the MS level (Figure 5).

Given the faint optical counterparts of A1 and A2 (see discussion in Section 3), these sources were not part of the S16 red sample. Their colors (Figure 2) and estimated IR luminosities (Figure 3) clearly point toward these being dusty star-forming sources, likely interacting/merging with their optically bright close neighbors (see also C18). These galaxies are shown as open symbols in Figure 5. We note that given the faintness and surroundings of both sources, we could not derive reliable stellar masses from multiband SED fitting as for the other galaxies in our sample. The stellar mass estimates shown in the figure for A1 and A2 are derived from dynamical masses inferred from the $\mathrm{CO}(4-3)$ resolved emission and measured line widths, under the assumptions detailed in C18. As discussed in $\mathrm{C} 18$, at least for A1 this estimate is consistent with a stellar mass derived empirically from the F105WF140W colors and F140W magnitudes as described above.

Figure 5 thus shows our current best picture of the massive $\left(\log \left(M / M_{\odot}\right)>10.5\right)$ cluster galaxy population in the central

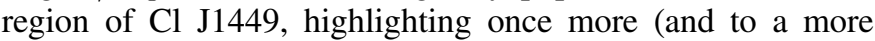
significant degree than our previous work; e.g., Gobat et al. 2011, 2013; Strazzullo et al. 2013, 2016) its composite nature combining very actively star-forming galaxies and sources with already-suppressed star formation.

\section{A Panchromatic Snapshot of a Forming Brightest Cluster Galaxy at $z=2$}

Figure 6 highlights the complex of multiple, likely interacting components located close to the center of the extended X-ray emission and galaxy overdensity in Cl J1449, identified as the forming cluster proto-BCG (Gobat et al. 2011), and including sources $\mathrm{H} 1, \mathrm{H} 4, \mathrm{H} 5$, and $\mathrm{A} 1$. We note that although other cluster members $(\mathrm{H} 2, \mathrm{H} 6)$ have stellar masses consistent with the individual masses of $\mathrm{H} 1, \mathrm{H} 4$, and $\mathrm{H} 5$ (see S16 and Figure 5), the configuration of the H1, H4, H5, A1 complex discussed below, as well as its location with respect to the galaxy overdensity and X-ray emission, is clearly much more suggestive, as compared to $\mathrm{H} 2$ or $\mathrm{H} 6$, of the site of main formation of the future cluster BCG. We stress for clarity that we identify this galaxy complex as a whole as the forming proto- $B C G$ and that we do not observe in $\mathrm{Cl} \mathrm{J} 1449$ any galaxy already exhibiting the peculiar features of BCGs.

Within a radius of $r=1$ !.5 we identify the two massive quiescent sources $\mathrm{H} 4$ and H5, the massive star-forming galaxy H1 with potentially sub-MS star formation activity, and the 

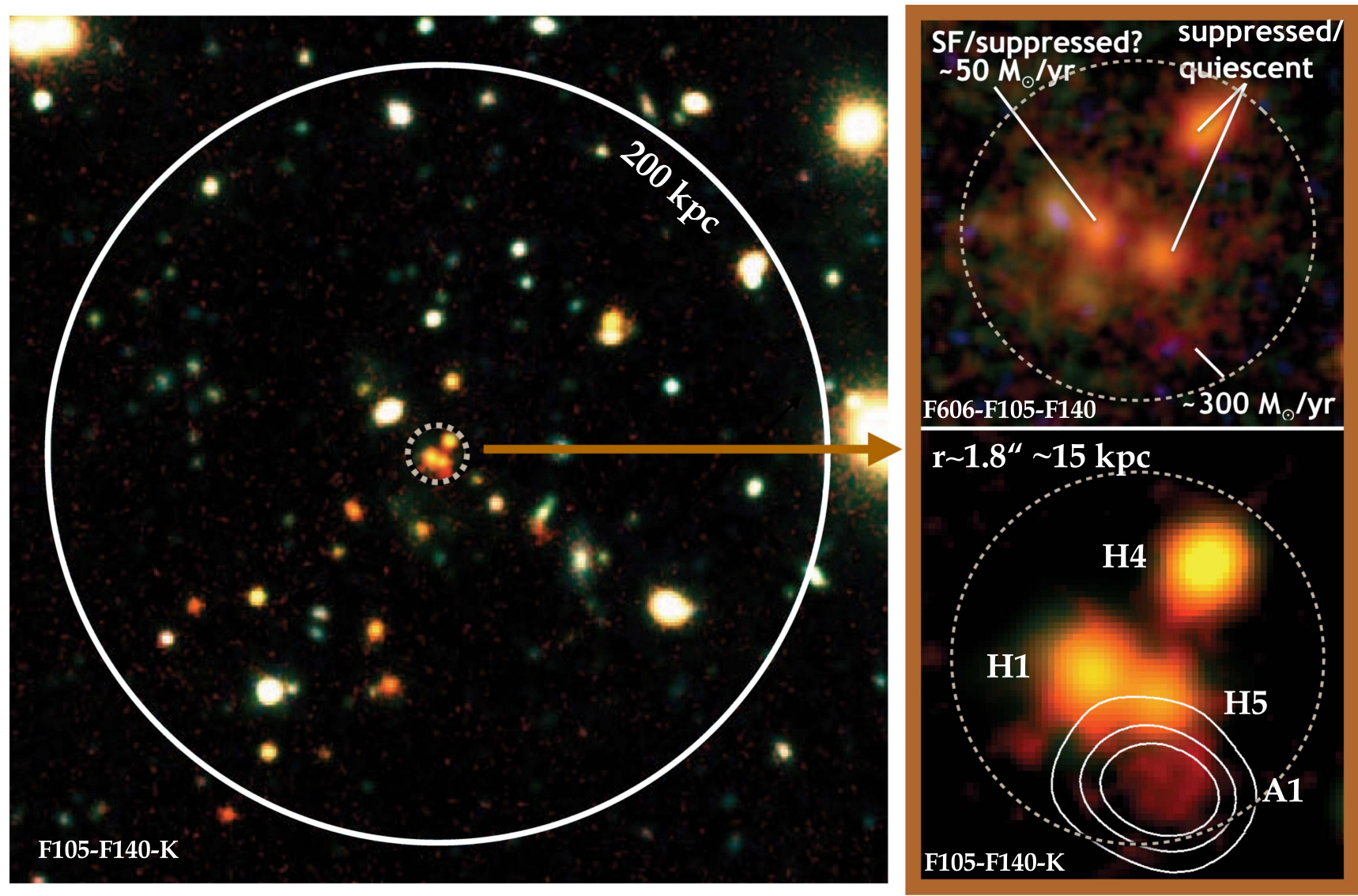

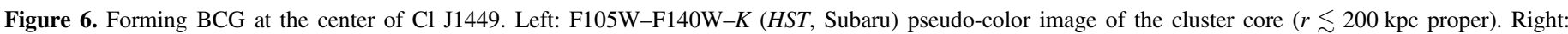

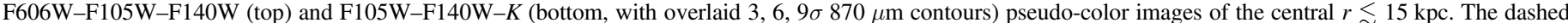

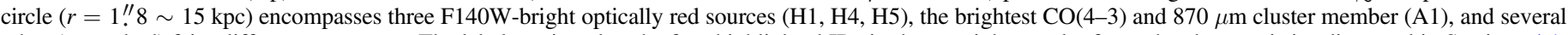

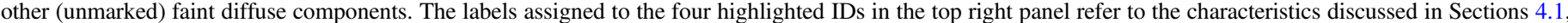
4.2 , and 5 .

optically faint and very red, millimeter-brightest cluster member A1, which is forming stars at a few hundred solar masses per year (Sections 3, 4.1, and 4.2). Indeed, Spitzer/ MIPS $24 \mu \mathrm{m}$ imaging already suggested this level of star formation associated with the proto-BCG (with obvious uncertainties related to the probed rest-frame wavelength and poor angular resolution; Gobat et al. 2011). The combination of HST color imaging and ALMA observations proves that the bulk of the star formation in the proto-BCG is actually occurring in the optically faint, seemingly minor component A1, rather than in the optically bright source $\mathrm{H} 1$ as originally thought (Gobat et al. 2013; Strazzullo et al. 2013). Additional faint components and tails, whose cluster membership and properties cannot be reliably determined with the current observations, are observed within $r \lesssim 1$ !' 5 from the proto-BCG (Figure 6).

These observations are strongly suggestive of an actively forming BCG still assembling its stellar mass through star formation and merging. This is in line with results from the few first studies on distant BCGs, suggesting a strong increase in the fraction of highly star-forming systems (Webb et al. 2015b; McDonald et al. 2016; Bonaventura et al. 2017), with $L_{\mathrm{IR}}>10^{12} L_{\odot}$ sources likely approaching $\gtrsim 50 \%$ of BCGs toward $z \sim 2$. As also discussed in previous work (Webb et al. 2015a, 2015b; Kubo et al. 2016; Bonaventura et al. 2017), gas-rich mergers might play a significant role in this phase of $\mathrm{BCG}$ evolution, as suggested by our observations as well (see also the related discussion on merger-driven star formation in Cl J1449 at large in Coogan et al. 2018). Besides the ongoing star formation activity, we stress, though, that a significant fraction of the stellar mass in the proto-BCG complex has already evolved to a seemingly quiescent phase. The depletion timescale estimated in $\mathrm{C} 18$ for the most actively star-forming component A1 is of order $100 \mathrm{Myr}$. The overall picture is qualitatively reminiscent of hierarchical model renditions (e.g., De Lucia \& Blaizot 2007; Tonini et al. 2012), though the observations presented here alone are obviously not sufficient to discuss the details of such modeling. Given the estimated (baryonic) masses of $\mathrm{A} 1, \mathrm{H} 1, \mathrm{H} 4$, and $\mathrm{H} 5$ and their relative projected distance, an approximate estimate of the merger timescale would be of order a few hundred million years (e.g., Lotz et al. 2011, the orbital timescale giving a lower limit of $\sim 100 \mathrm{Myr}$ ), with an estimated total stellar mass of the resulting $\mathrm{BCG} \sim 3 \times 10^{11} M_{\odot}$.

\section{Summary}

ALMA observations of the $870 \mu$ m continuum and $\mathrm{CO}(4-3)$ line emission in the central region of $\mathrm{Cl} \mathrm{J} 1449$ have significantly improved our understanding of galaxy populations in this cluster core. Crucially, $\mathrm{CO}(4-3)$ follow-up secured spectroscopic confirmation of optically faint, millimeter-bright cluster members, while questioning the membership of the two brightest $870 \mu \mathrm{m}$ sources in the field. 
The $870 \mu \mathrm{m}$ continuum and $\mathrm{CO}(4-3)$ line emission yield a total estimated SFR within the probed $\sim 0.08 \mathrm{Mpc}^{2}$ region of $\sim 700 \pm 100 M_{\odot} \mathrm{yr}^{-1}$, resulting in a projected SFR density of $\sim(0.9 \pm 0.1) \times 10^{4} \mathrm{M}_{\odot} \mathrm{yr}^{-1} \mathrm{Mpc}^{-2}$ and a SFR volume density five orders of magnitude larger than in the field at the same redshift. The inferred lower limit (that is, not correcting for the missing SFR from the portion of the virial volume not probed by the ALMA observations) on the SFR density per halo mass is $\mathrm{SFR} / M_{\text {halo }} \gtrsim 1300 \pm 400 M_{\odot} \mathrm{yr}^{-1} / 10^{14} M_{\odot}$, which at face value is consistent with extrapolations from lower-redshift observations predicting high SFR densities in massive (yet sub-10 ${ }^{14} M_{\odot}$ ) halos at this redshift (see discussion in Section 4.1). In spite of its relatively significant SFR density, the core of $\mathrm{Cl} \mathrm{J} 1449$ seems nonetheless far from the $>3000 M_{\odot} \mathrm{yr}^{-1}$ observed within a similar (or smaller) clustercentric distance in the similarly massive $\mathrm{Cl} \mathrm{J1001+0220} \mathrm{at}$ $z=2.5$ (Wang et al. 2016). Three of the five most massive galaxies $\left(\mathrm{H} 2, \mathrm{H} 4, \mathrm{H} 5 ; \log \left(M / M_{\odot}\right) \sim 11 \pm 0.1\right)$ in the core of Cl J1449 are seemingly quiescent sources remaining undetected nominally at least $2 \sigma$ below the MS (the other two being the MS star-forming galaxy H6 and the possibly suppressed H1; see Section 4.2, Figure 5), compared to a star-forming galaxy fraction of $80 \%$ observed at $M \geqslant 10^{11} M_{\odot}$ in the core of Cl J1001+0220 (Wang et al. 2016).

The combination with previously available HST imaging (and grism spectroscopy) critically enhances the interpretation of these observations. Although there is generally a close correspondence between the millimeter- and optical/NIRinferred pictures of most cluster galaxies discussed here, there are particular sources that can only be really understood by comparing the two. The bright ALMA sources A1 and A2 are associated with optical counterparts otherwise deemed comparatively minor components in the HST NIR imaging. Figure 2 underlines that, if deep high-resolution color imaging were not available, these ALMA detections would appear as dust and gas significantly offset from stellar emission in the nearest optical/NIR counterpart. The HST imaging reveals instead the faint, extremely red components perfectly matching the submillimeter emission, and probably related to very recent or ongoing merging events (see further discussion in C18).

The HST and ALMA synergy also provides in this cluster another striking snapshot of the early evolution of forming BCGs (e.g., Webb et al. 2015a; Kubo et al. 2016, see Section 5), with a seemingly multiple-merger system of quiescent and highly star-forming components likely assembling the future BCG.

The results based on ALMA observations presented here extend the reach of our previous studies in Cl J1449, drawing quantitative details in a picture combining, right into the cluster core, a star formation activity approaching a thousand solar masses per year, the first massive quiescent cluster-core galaxies, and the ongoing formation of the BCG through merging of already quiescent and still vigorously star-forming components.

Largely based on observations from ALMA programs 2012.1.00885.S and 2015.1.01355.S and HST programs GO11648 and GO-12991. We thank the anonymous referee for a constructive report improving the presentation of this work. R.T.C. acknowledges support from STFC G1687 grant ST/ N504452/1. F.V. acknowledges the Villum Fonden research grant 13160 "Gas to Stars, Stars to Dust: Tracing Star Formation across Cosmic Time.” A.R. acknowledges support by INAF grant PRIN-2017. N.A. is supported by the Brain Pool Program, which is funded by the Ministry of Science and ICT through the National Research Foundation of Korea (2018H1D3A2000902). A.C. acknowledges PRIN MIUR 2015 "Cosmology and Fundamental Physics: Illuminating the Dark Universe with Euclid." H.D. acknowledges financial support from the Spanish Ministry of Economy and Competitiveness (MINECO) under the 2014 Ramón y Cajal program MINECO RYC-2014-15686. This paper makes use of the following ALMA data: ADS/JAO.ALMA\#2012.1.00885.S, ADS/JAO. ALMA\#2015.1.01355.S. ALMA is a partnership of ESO (representing its member states), NSF (USA) and NINS (Japan), together with NRC (Canada), MOST and ASIAA (Taiwan), and KASI (Republic of Korea), in cooperation with the Republic of Chile. The Joint ALMA Observatory is operated by ESO, AUI/NRAO and NAOJ. Partly based on observations made with the NASA/ESA Hubble Space Telescope, obtained from the Data Archive at the Space Telescope Science Institute, which is operated by the Association of Universities for Research in Astronomy, Inc., under NASA contract NAS 5-26555.

\section{ORCID iDs}

V. Strazzullo (ib https://orcid.org/0000-0001-7975-2894

E. Daddi (iD https://orcid.org/0000-0002-3331-9590

M. T. Sargent (i) https://orcid.org/0000-0003-1033-9684

R. Gobat (iD https://orcid.org/0000-0003-0121-6113

F. Valentino (iD https://orcid.org/0000-0001-6477-4011

M. Pannella (1) https://orcid.org/0000-0003-3738-3976

A. Renzini (1) https://orcid.org/0000-0002-7093-7355

A. Cimatti (i) https://orcid.org/0000-0002-4409-5633

H. Dannerbauer (i) https://orcid.org/0000-0001-7147-3575

A. Finoguenov (1) https://orcid.org/0000-0002-4606-5403

M. Onodera (ib https://orcid.org/0000-0003-3228-7264

\section{References}

Alavi, A., Siana, B., Richard, J., et al. 2016, ApJ, 832, 56

Alberts, S., Pope, A., Brodwin, M., et al. 2014, MNRAS, 437, 437

Alberts, S., Pope, A., Brodwin, M., et al. 2016, ApJ, 825, 72

Andreon, S. 2006, A\&A, 448, 447

Andreon, S., Dong, H., \& Raichoor, A. 2016, A\&A, 593, A2

Andreon, S., Maughan, B., Trinchieri, G., \& Kurk, J. 2009, A\&A, 507, 147

Andreon, S., Newman, A. B., Trinchieri, G., et al. 2014, A\&A, 565, A120

Aravena, M., Carilli, C. L., Salvato, M., et al. 2012, MNRAS, 426, 258

Beifiori, A., Mendel, J. T., Chan, J. C. C., et al. 2017, ApJ, 846, 120

Béthermin, M., Daddi, E., Magdis, G., et al. 2012, ApJL, 757, L23

Bleem, L. E., Stalder, B., de Haan, T., et al. 2015, ApJS, 216, 27

Bonaventura, N. R., Webb, T. M. A., Muzzin, A., et al. 2017, MNRAS, 469, 1259

Bothwell, M. S., Smail, I., Chapman, S. C., et al. 2013, MNRAS, 429, 3047 Brodwin, M., Stanford, S. A., Gonzalez, A. H., et al. 2013, ApJ, 779, 138 Bruzual, G., \& Charlot, S. 2003, MNRAS, 344, 1000

Cai, Z., Fan, X., Bian, F., et al. 2017, ApJ, 839, 131

Calzetti, D., Armus, L., Bohlin, R. C., et al. 2000, ApJ, 533, 682

Casasola, V., Magrini, L., Combes, F., et al. 2013, A\&A, 558, A60 Casey, C. M. 2016, ApJ, 824, 36

Casey, C. M., Cooray, A., Capak, P., et al. 2015, ApJL, 808, L33 Chan, J. C. C., Beifiori, A., Saglia, R. P., et al. 2018, ApJ, 856, 8 Clements, D. L., Braglia, F. G., Hyde, A. K., et al. 2014, MNRAS, 439, 1193 Coogan, R., Daddi, E., Sargent, M. T., et al. 2018, MNRAS, 479, 703

Cooke, E. A., Hatch, N. A., Muldrew, S. I., Rigby, E. E., \& Kurk, J. D. 2014, MNRAS, 440, 3262

Cooke, E. A., Hatch, N. A., Stern, D., et al. 2016, ApJ, 816, 83

Daddi, E., Dannerbauer, H., Liu, D., et al. 2015, A\&A, 577, A46

Daddi, E., Jin, S., Strazzullo, V., et al. 2017, ApJL, 846, L31

Dannerbauer, H., Kurk, J. D., De Breuck, C., et al. 2014, A\&A, 570, A55

Dannerbauer, H., Lehnert, M. D., Emonts, B., et al. 2017, A\&A, 608, A48 
De Lucia, G., \& Blaizot, J. 2007, MNRAS, 375, 2

De Propris, R., Stanford, S. A., Eisenhardt, P. R., Holden, B. P., \& Rosati, P. 2007, AJ, 133, 2209

Eisenhardt, P. R. M., Brodwin, M., Gonzalez, A. H., et al. 2008, ApJ, 684, 905 Elbaz, D., Dickinson, M., Hwang, H. S., et al. 2011, A\&A, 533, A119

Emonts, B. H. C., Feain, I., Röttgering, H. J. A., et al. 2013, MNRAS, 430, 3465

Fixsen, D. J., Bennett, C. L., \& Mather, J. C. 1999, ApJ, 526, 207

Flores-Cacho, I., Pierini, D., Soucail, G., et al. 2016, A\&A, 585, A54

Geach, J. E., Smail, I., Ellis, R. S., et al. 2006, ApJ, 649, 661

Gobat, R., Daddi, E., Magdis, G., et al. 2018, NatAs, 2, 239

Gobat, R., Daddi, E., Onodera, M., et al. 2011, A\&A, 526, A133

Gobat, R., Strazzullo, V., Daddi, E., et al. 2013, ApJ, 776, 9

Granato, G. L., Ragone-Figueroa, C., Domínguez-Tenreiro, R., et al. 2015, MNRAS, 450, 1320

Gullberg, B., Lehnert, M. D., De Breuck, C., et al. 2016, A\&A, 591, A73

Guo, Y., Ferguson, H. C., Giavalisco, M., et al. 2013, ApJS, 207, 24

Hatch, N. A., Kurk, J. D., Pentericci, L., et al. 2011, MNRAS, 415, 2993

Hatch, N. A., Muldrew, S. I., Cooke, E. A., et al. 2016, MNRAS, 459, 387

Hayashi, M., Kodama, T., Kohno, K., et al. 2017, ApJL, 841, L21

Hayashi, M., Kodama, T., Koyama, Y., et al. 2010, MNRAS, 402, 1980

Hayashi, M., Kodama, T., Koyama, Y., Tadaki, K.-I., \& Tanaka, I. 2011, MNRAS, 415, 2670

Hayashi, M., Kodama, T., Tanaka, I., et al. 2016, ApJL, 826, L28

Hayashi, M., Tadaki, K.-i., Kodama, T., et al. 2018, ApJ, 856, 118

Hilton, M., Lloyd-Davies, E., Stanford, S. A., et al. 2010, ApJ, 718, 133

Hung, C.-L., Casey, C. M., Chiang, Y.-K., et al. 2016, ApJ, 826, 130

Husband, K., Bremer, M. N., Stott, J. P., \& Murphy, D. N. A. 2016, MNRAS, 462,421

Ivison, R. J., Swinbank, A. M., Smail, I., et al. 2013, ApJ, 772, 137

Kacprzak, G. G., Yuan, T., Nanayakkara, T., et al. 2015, ApJL, 802, L26

Kennicutt, R. C., Jr. 1998, ARA\&A, 36, 189

Kewley, L. J., Yuan, T., Nanayakkara, T., et al. 2016, ApJ, 819, 100

Koyama, Y., Kodama, T., Tadaki, K.-i., et al. 2014, ApJ, 789, 18

Koyama, Y., Smail, I., Kurk, J., et al. 2013, MNRAS, 434, 423

Krishnan, C., Hatch, N. A., Almaini, O., et al. 2017, MNRAS, 470, 2170

Kubo, M., Uchimoto, Y. K., Yamada, T., et al. 2013, ApJ, 778, 170

Kubo, M., Yamada, T., Ichikawa, T., et al. 2016, MNRAS, 455, 3333

Kulas, K. R., McLean, I. S., Shapley, A. E., et al. 2013, ApJ, 774, 130

Kurk, J., Cimatti, A., Zamorani, G., et al. 2009, A\&A, 504, 331

Kurk, J. D., Röttgering, H. J. A., Pentericci, L., et al. 2000, A\&A, 358, L1

Lee, M. M., Tanaka, I., Kawabe, R., et al. 2017, ApJ, 842, 55

Lee-Brown, D. B., Rudnick, G. H., Momcheva, I. G., et al. 2017, ApJ, 844, 43

Lidman, C., Rosati, P., Tanaka, M., et al. 2008, A\&A, 489, 981

Liu, D., Gao, Y., Isaak, K., et al. 2015, ApJL, 810, L14

Lotz, J. M., Jonsson, P., Cox, T. J., et al. 2011, ApJ, 742, 103

Lotz, J. M., Papovich, C., Faber, S. M., et al. 2013, ApJ, 773, 154

Ma, C.-J., Smail, I., Swinbank, A. M., et al. 2015, ApJ, 806, 257

Madau, P., \& Dickinson, M. 2014, ARA\&A, 52, 415

Mancone, C. L., Gonzalez, A. H., Brodwin, M., et al. 2010, ApJ, 720, 284

Mantz, A. B., Abdulla, Z., Allen, S. W., et al. 2018, A\&A, in press (arXiv:1703.08221)

McDonald, M., Stalder, B., Bayliss, M., et al. 2016, ApJ, 817, 86

Mei, S., Holden, B. P., Blakeslee, J. P., et al. 2009, ApJ, 690, 42

Mullis, C. R., Rosati, P., Lamer, G., et al. 2005, ApJL, 623, L85

Muzzin, A., Wilson, G., Demarco, R., et al. 2013, ApJ, 767, 39
Nantais, J. B., Muzzin, A., van der Burg, R. F. J., et al. 2017, MNRAS, 465, L104

Navarro, J. F., Frenk, C. S., \& White, S. D. M. 1997, ApJ, 490, 493

Newman, A. B., Ellis, R. S., Andreon, S., et al. 2014, ApJ, 788, 51

Noble, A. G., McDonald, M., Muzzin, A., et al. 2017, ApJL, 842, L21

Oteo, I., Zwaan, M. A., Ivison, R. J., Smail, I., \& Biggs, A. D. 2016, ApJ, 822,36

Overzier, R. A. 2016, A\&ARv, 24, 14

Pannella, M., Elbaz, D., Daddi, E., et al. 2015, ApJ, 807, 141

Papadopoulos, P. P., van der Werf, P. P., Xilouris, E. M., et al. 2012, MNRAS, 426, 2601

Papovich, C., Momcheva, I., Willmer, C. N. A., et al. 2010, ApJ, 716, 1503

Parsa, S., Dunlop, J. S., McLure, R. J., \& Mortlock, A. 2016, MNRAS, 456,3194

Popesso, P., Biviano, A., Finoguenov, A., et al. 2015a, A\&A, 574, A105

Popesso, P., Biviano, A., Finoguenov, A., et al. 2015b, A\&A, 579, A132

Prichard, L. J., Davies, R. L., Beifiori, A., et al. 2017, ApJ, 850, 203

Rosati, P., Stanford, S. A., Eisenhardt, P. R., et al. 1999, AJ, 118, 76

Rudnick, G., Hodge, J., Walter, F., et al. 2017, ApJ, 849, 27

Salpeter, E. E. 1955, ApJ, 121, 161

Santos, J. S., Altieri, B., Valtchanov, I., et al. 2015, MNRAS, 447, L65

Santos, J. S., Fassbender, R., Nastasi, A., et al. 2011, A\&A, 531, L15

Sargent, M. T., Daddi, E., Béthermin, M., et al. 2014, ApJ, 793, 19

Schreiber, C., Pannella, M., Elbaz, D., et al. 2015, A\&A, 575, A74

Shimakawa, R., Kodama, T., Hayashi, M., et al. 2018, MNRAS, 473, 1977

Shimakawa, R., Kodama, T., Tadaki, K.-i., et al. 2015, MNRAS, 448, 666

Smail, I., Geach, J. E., Swinbank, A. M., et al. 2014, ApJ, 782, 19

Snyder, G. F., Brodwin, M., Mancone, C. M., et al. 2012, ApJ, 756, 114

Spitler, L. R., Labbé, I., Glazebrook, K., et al. 2012, ApJL, 748, L21

Stach, S. M., Swinbank, A. M., Smail, I., et al. 2017, ApJ, 849, 154

Stanford, S. A., Brodwin, M., Gonzalez, A. H., et al. 2012, ApJ, 753, 164

Stanford, S. A., Romer, A. K., Sabirli, K., et al. 2006, ApJL, 646, L13

Steidel, C. C., Adelberger, K. L., Shapley, A. E., et al. 2005, ApJ, 626, 44

Strazzullo, V., Daddi, E., Gobat, R., et al. 2015, A\&A, 576, L6

Strazzullo, V., Daddi, E., Gobat, R., et al. 2016, ApJL, 833, L20

Strazzullo, V., Gobat, R., Daddi, E., et al. 2013, ApJ, 772, 118

Strazzullo, V., Rosati, P., Pannella, M., et al. 2010, A\&A, 524, A17

Tadaki, K.-i., Kodama, T., Tamura, Y., et al. 2014, ApJL, 788, L23

Tanaka, M., Finoguenov, A., \& Ueda, Y. 2010, ApJL, 716, L152

Tanaka, M., Toft, S., Marchesini, D., et al. 2013, ApJ, 772, 113

Tonini, C., Bernyk, M., Croton, D., Maraston, C., \& Thomas, D. 2012, ApJ, 759,43

Tran, K.-V. H., Nanayakkara, T., Yuan, T., et al. 2015, ApJ, 811, 28

Valentino, F., Daddi, E., Finoguenov, A., et al. 2016, ApJ, 829, 53

Valentino, F., Daddi, E., Strazzullo, V., et al. 2015, ApJ, 801, 132

Venemans, B. P., Röttgering, H. J. A., Miley, G. K., et al. 2007, A\&A, 461,823

Wang, T., Elbaz, D., Daddi, E., et al. 2016, ApJ, 828, 56

Webb, T., Noble, A., DeGroot, A., et al. 2015a, ApJ, 809, 173

Webb, T. M. A., Muzzin, A., Noble, A., et al. 2015b, ApJ, 814, 96

Webb, T. M. A., O'Donnell, D., Yee, H. K. C., et al. 2013, AJ, 146, 84

Williams, R. J., Quadri, R. F., Franx, M., et al. 2009, ApJ, 691, 1879

Wilson, G., Muzzin, A., Yee, H. K. C., et al. 2009, ApJ, 698, 1943

Wylezalek, D., Galametz, A., Stern, D., et al. 2013, ApJ, 769, 79

Wylezalek, D., Vernet, J., De Breuck, C., et al. 2014, ApJ, 786, 17

Zeimann, G. R., Stanford, S. A., Brodwin, M., et al. 2012, ApJ, 756, 115 